\title{
METHODS FOR ESTIMATING VEHICLE QUEUES AT A MARINE TERMINAL: A COMPUTATIONAL COMPARISON
}

\author{
GANG CHEN, ZHONG-ZHEN YANG
}

Transport Planning Institute

Dalian Maritime University, Linghai Road 1, Dalian, Liaoning Province, PR China

e-mail: clenchen@gmail.com

\begin{abstract}
A long queue of vehicles at the gate of a marine terminal is a common traffic phenomenon in a port-city, which sometimes causes problems in urban traffic. In order to be able to solve this issue, we firstly need accurate models to estimate such a vehicle queue length. In this paper, we compare the existing methods in a case study, and evaluate their advantages and disadvantages. Particularly, we develop a simulation-based regression model, using the micro traffic simulation software PARAMIC. In simulation, it is found that the queue transient process follows a natural logarithm curve. Then, based on these curves, we develop a queue length estimation model. In the numerical experiment, the proposed model exhibits better estimation accuracy than the other existing methods.
\end{abstract}

Keywords: truck queue, container terminal, queueing theory, simulation, regression.

\section{Introduction}

A long truck queue is a common problem at large-scale transportation and logistics facilities, for example, seaports, logistics terminals and airports. Typically, these high investment facilities have relatively stable capacities. Due to the expensive and limited resources, including land, it is not always possible to expand and to increase supply after completion. On the other hand, customer demand does not evenly distribute over time. So customer queues occur very often at peak times, which in turn limit service capacity. In such a case, management of customer arrivals can be used to reduce congestion and utilize existing facilities/equipment. We believe that the need for demand management will be common for these high investment and large-scale transportation facilities, by virtue of the popularization of information technology. Before being able to apply any demand management, we need good prediction models to estimate the queue length with high accuracy.

A marine terminal is a typical example of the above-mentioned facilities. As a consequence of the continually increasing cargo traffic volume and the introduction of mega-ships, truck congestion at terminal gates becomes a common problem in many major seaports. Truck queues generate serious air pollution and limit the capacities of truck fleets and terminal systems.
It is in the interest of both public and private sectors to reduce the queues and maintain the efficiency of freight flows. However, in the literature, we have not seen a comparison study on existing truck queue length estimation methods that would show which one is suitable in what situation.

Generally, the gate system of a marine terminal is in a dynamic situation, where both the truck arrival rate and the gate service rate are variable over time. The intervals of such changes are too short for a nearly saturated queue to reach its steady state. As shown in Fig. 1, it takes time for a queue to reach the steady state, and both the formation process and the steady state play important roles in analysing the queue. Moreover, truck queues have apparent characteristic of congested traffic. Therefore, to accurately estimate the queue length of trucks at the gate in a marine terminal, it is necessary to capture the transient behaviour of a dynamic queue and also the traffic characteristic of vehicle queues.

In the literature, we can find five methods to estimate the truck queue length at a marine terminal gate. They are the fluid flow model, stationary queueing models, non-stationary queueing models, simulation models and regression models. This paper tries to compare these methods by applying all of them to one case, so that we can see the advantages and disadvantages of each method in a real case application. Specifically, for 


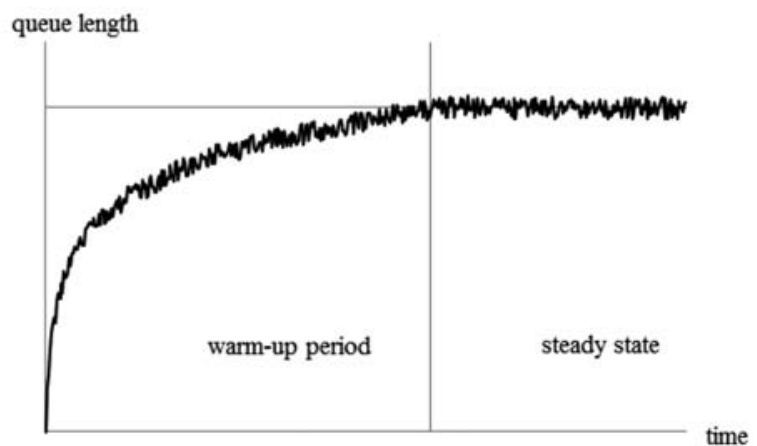

Fig. 1. Transient behaviour of a queue simulation with an idle initial condition.

the regression method, in this study we develop a simulation-based regression model using data from a micro traffic simulation, where detailed vehicle driving behaviour is well captured. This model is good at the curving vehicle queue formation process, and has higher estimation accuracy compared with the other methods in a numerical experiment.

The rest of this paper is organized as follows. Section 2 provides a literature review on the existing studies on truck queue estimation. Section 3 gives a detailed model of each estimation method of the truck queue at a terminal gate (except for the regression method). Section 4 describes the development of the proposed simulation-based regression model. Section 5 shows a case study where all the methods are compared in terms of estimation accuracy and advantages, and concluding remarks are provided in Section 6.

\section{Literature review}

In the existing studies, there are five types of vehicle queue estimation methods for the gate system of a large-scale transportation and logistics facility. In this section, we review these methods one by one.

The first one is the fluid flow method, which basically follows the flow balance principle, meaning the change in a queue equals flow-in minus flow-out. This principle holds only in a deterministic environment, but when we estimate an average queue length in a stochastic environment, it is always underestimated, because it simply neglects the queuing phenomenon caused by stochastic elements in arrival and service processes. For example, the model by Chen and Yang (2010) simply assumes that no queue happens when the arrival rate is lower than the gate capacity. Their model definitely underestimates truck queue lengths, because queuing theory says that a queue can always happen even when the arrival rate does not exceed the server capacity within a period.
The second method is stationary queuing models, which have been widely applied in different industries, for example, Internet traffic management (Chydziński and Chróst, 2011), service industries (Davidson, 1988) and logistics industry (Guan and Liu, 2009; Kim, 2009; Taniguchi et al., 1999). Guan and Liu (2009) use a multi-server $M / E_{k} / s$ queuing model to analyse the gate queue at one container terminal in the port of New York/New Jersey. They neglect the queue transient/growing process and assume that a queue can reach its steady state instantaneously. Therefore, the queue lengths in their study might be very likely overestimated. Taniguchi et al. (1999) also apply an $M / E_{k} / s$ queuing model to explain the truck movements in a public logistic terminal. A similar queuing system also exists in some other research areas, for example, the toll plaza design/optimization problem, which has a similar structure and functions as a terminal gate. Kim (2009) uses an $M / G / 1$ queueing model to analyse the car queues in a toll plaza. As discussed by Srivatsan and Kempf (1995), the steady state assumptions preclude use of short-term throughput time predictions. Accordingly a common problem of these applications is that, due to neglecting the queue transient process, these stationary queueing models probably lead to overcapacity design of the facility. Furthermore, stationary queueing models cannot handle situations where demand is over capacity, which also often occurs at transportation and logistics facilities.

The third method is to adopt non-stationary queueing models. Chen et al. (2011) develop a two-layer queuing network, in which the gate system is treated as multiple independent $M(t) / M(t) / 1$ queues (each gate lane is an $M(t) / M(t) / 1$ queue) and the yard system is treated as multiple independent non-stationary $M(t) / G(t) / 1$ queues (each yard zone is an $M(t) / G(t) / 1$ queue). This queuing network can model the truck queues at gate lanes and in yard zones jointly. However, there are two limitations of this queuing network: first, the assumption that the gate service follows an exponential distribution is not based on any empirical study; second, the assumption that a gate system comprises multiple independent queuing systems does not fit practice, as truckers always choose a short lane to wait, which means the gate system should be viewed as a single queuing system with multiple servers. Chen et al. (2013) develop a fluid-based approximation for a non-stationary exponential Erlang queuing system, called the B-PSFFA approximation method. They apply the B-PSFFA approximation to analyse the truck queue length at the gate of a container terminal and compare it with the stationary model in Guan and Liu (2009). Taking the simulation result of 10,000 replications as the exact estimation, the comparison result shows that the stationary queuing model is inaccurate, while the B-PSFFA approximation of a non-stationary 
queue can reach high accuracy.

The fourth method is to conduct a traffic simulation study. Especially, a micro level traffic simulation can capture the traffic characteristic of vehicle queues (Wu et al., 2013). Specifically, for a terminal gate system, simulation can curve not only the queue transient process in a dynamic situation, but also the detailed vehicle driving behaviour. In recent years, simulation has been widely used in container terminal studies. Recent examples include the results of Kia et al. (2002), Liu et al. (2002), Shabayek and Yeung (2002), Yang et al. (2004), Hartmann (2004) as well as Sacone and Siri (2009). There are many traffic simulation software options available on the market, for example, the PARAMICS microscopic simulation package (Xie et al., 2012). On the other hand, like any type of simulation, traffic simulation could be a time-consuming study.

The fifth method is to develop an analytical model. Huynh et al. (2004) develop a discrete event simulation model of a container terminal with the Arena simulation language, which can present the movements of trucks and yard cranes. The simulation data for all the scenarios is analysed with regression. Based on the regression results, they develop a truck turn time estimation model with the independent variable of the average truck number served by a crane. However, their model covers only the yard part of a marine terminal, rather than the gate part.

Based on the above literature review, we can see that there are some existing methods for estimating the truck queue length at a marine terminal, but there was no comprehensive comparison study on the efficiency and the accuracy of these methods. This paper tries to make such a comparison and also aims at developing an efficient and accurate queue length estimation model for the gate system in a marine terminal. The adopted method is a combination of microscopic traffic simulation and regression approach.

\section{Existing methods}

In this section, we will introduce a specific model of each estimation method of the truck queue length at a terminal gate, except for the regression method.

3.1. Fluid flow model. Fluid flow models have a basic flow balance function, meaning the change in a queue length equals flow-in minus flow-out. The model used by Chen and Yang (2010) is

$$
l_{t}=\max \left\{0, l_{t-1}+\lambda_{t}-\mu\right\},
$$

where $l$ is the average queue length in the steady state, $t$ is the time interval, $\lambda$ is the average truck arrival rate per time interval, and $\mu$ is the average gate service rate per time interval.
3.2. Stationary queueing model. The stationary queuing model is a classical tool to approximate a queuing system/network, and allows a number of useful steady state performance measures to be determined. It has been widely applied in different fields, e.g., communication networks, computer systems, machine plants, and so forth. At the same time, it has also received some critical comments; the major one is that the assumptions are too restrictive to be able to model real-world situations exactly.

Based on the physical layout and the characteristics, a container terminal gate system can be treated as a multi-server queuing system with multiple inbound and outbound lanes, so a multi-server queuing model is applicable to analyse the steady state of a truck queue. A multi-server queuing model has the following additional parameters: $s$ as the number of gate lanes and $W$ as the average customer waiting time in the steady state.

The type of queueing model is determined by the statistical distributions of truck arrival intervals and gate service time. Two existing studies, by Guan and Liu (2009) as well as Taniguchi et al. (1999), adopt the type $M / E_{k} / s$ of queuing models to explain the truck movements in a marine container terminal and a public logistic terminal, while a study in the neighbour field of toll plaza optimization by Kim (2009) used an $M / G / 1$ queueing model. Our comment on the above model types is that Guan and Liu (2009) as well as Taniguchi et al. (1999) mixed the empty and loaded trucks together in the queue analysis and concluded an Erlang distribution of the service time for both kinds of trucks. For several reasons, however, it is typical to divide the lane services for empty and loaded trucks in a container terminal. The average service time for empty trucks is normally much lower than the one for loaded trucks. It is acceptable to assume the service times for empty/loaded trucks following a general distribution (Tianjin Port, personal conversations). Therefore, in our problem, the gate system is modeled as an $M / G / s$ queueing model, where the arrival process follows a Poisson distribution (meaning the truck inter-arrival times follow an exponential distribution) and the gate service times follow a general distribution.

Since no conventional mathematical $M / G / s$ is available, approximation is necessary. The well-known approximation formula was developed by Cosmetatos (1976). This approximation relates the average waiting time of trucks in an $M / G / s$ model to that in an $M / M / s$ model. The system utilization factor $\rho$ and the average waiting time of trucks $W^{\prime}$ are respectively given by

$$
\rho=\frac{\lambda}{\mu}
$$




$$
\begin{aligned}
W^{\prime}= & \frac{\rho^{s}}{\mu(s-1) !(s-\rho)^{2}} \\
& \times\left\{\sum_{n=0}^{s-1} \frac{\rho^{n}}{n !}+\frac{\rho^{s}}{(s-1) !(s-\rho)}\right\} .
\end{aligned}
$$

The Cosmetatos approximation formula is given

$$
\begin{aligned}
W \cong & W^{\prime}\left\{\frac{1+v^{2}}{2}+\left(1-v^{2}\right)(1-\rho)\right. \\
& \left.\times(s-1) \frac{\sqrt{4+5 s}-2}{32 \rho s}\right\},
\end{aligned}
$$

where $v$ is the variation coefficient of gate service times in an $M / G / s$ model,

$$
v=\frac{\sqrt{v a r}}{\mu}
$$

in which var is the variation of gate service times. According to Little's law,

$$
L=W \lambda,
$$

the average queue length of $M / G / s$ model is

$$
\begin{aligned}
\mathrm{L} \cong & \mathrm{W} \times\left\{\frac{1+\mathrm{v}^{2}}{2}+\left(1-\mathrm{v}^{2}\right)(1-\rho)\right. \\
& \left.\times(\mathrm{s}-1) \frac{\sqrt{4+5 \mathrm{~s}}-2}{32 \rho \mathrm{s}}\right\} \lambda .
\end{aligned}
$$

3.3. Non-stationary queueing model. The limitation of stationary queueing models is that they neglect the transient behaviour of a queue and assume the queue can reach its static state instantaneously. Therefore, they are suitable only for queueing systems reaching the steady state. Typically the truck queues in a container terminal are not in steady state, because the truck arrival rates and gate service rates vary over time. To capture this kind of dynamic congestion, state-dependent queueing models are effective and robust tolls (Smith, 2010). Chen et al. (2011) develop a two-layer queuing network, in which the gate system is treated as multiple independent non-stationary $M(t) / M(t) / 1$ queues (each gate lane is an $M(t) / M(t) / 1$ queue) and the yard system is treated as multiple independent non-stationary $M(t) / G(t) / 1$ queues (each yard zone is an $M(t) / G(t) / 1$ queue). However, the assumption that the gate service times follow an exponential distribution is not based on any empirical study. In our problem, the gate service time is modelled with a general distribution, meaning the gate system can be treated as multiple independent non-stationary $M(t) / G(t) / 1$ queues (each gate lane is an $M(t) / G(t) / 1$ queue). Of course, there is an obvious limitation of assuming that a gate system comprises multiple independent queueing systems. But in the literature, there is no approximation to $M(t) / G(t) / s$ queues yet.
The detailed approximation for the $M(t) / G(t) / 1$ model developed by Chen et al. (2011) for truck queue length estimation is

$$
\begin{gathered}
l_{t}=l_{t-1}+\lambda_{t-1}-d_{t-1}, \quad \forall t, \\
\rho_{t}=\frac{l_{t}+1-\sqrt{\left(l_{t}\right)^{2}+2 \times l_{t} \times v^{2}+1}}{1-v^{2}}, \quad \forall t, \\
d_{t}=\min \left(l_{t}, \rho_{t} \mu_{t}\right), \quad \forall t .
\end{gathered}
$$

Equation (8) is a flow balance function, meaning that during a state transition the change queue length is equal to arrivals $\lambda_{t}$ minus departures $d_{t}$. Equation (9) derives the capacity utilization ratio $\rho_{t}$ based on the related queue length $l_{t}$ with $v$ being the variation coefficient of gate service times. Equation (10) determines the departure rate $d_{t}$ at time interval $t$ based on service capacity $\mu_{t}$ and the estimated capacity utilization ratio $\rho_{t}$. A note on this approximation is that the time interval has to be small enough so that the system (1) in an interval can be assumed as in steady state, and (2) $d_{t}$ at the first interval can be assumed zero.

3.4. Microscopic traffic simulation. Simulation is the process of designing a model of a real system and conducting experiments for the purpose of understanding the behaviour of the system. In our problem, truck queues have apparent traffic characteristics, so we adopt the micro simulation package PARAMICS developed by Quadstone Ltd. PARAMICS is a widely applicable micro simulation traffic flow modelling system-software for the analysis and design of urban and highway networks. It simulates the individual components of traffic flow and congestion, represents the actions and inter-actions of individual vehicles as they travel through a road network. In PARAMICS, there is a toll plaza function, which can model the physical structure of a terminal gate system. A screenshot of the simulation model is shown in Fig. 2.

\section{Simulation-based regression model}

In this section, we introduce the proposed simulation-based regression method for truck queue length estimation. The general framework, as shown in Fig. 3, includes the following four steps.

The first step is to develop a simulation model of the addressed queueing system with micro traffic simulation software. The simulation tool is PARAMICS, one of the best micro-traffic simulation applications, which can model detailed driving behaviour of every individual vehicle. We gather adequate queue data through simulation, by running the model for every possible scenario to cover both the formation process and the steady state of the truck queue. A good quality of 
simulation results can only be reached by hundreds of times of simulation replications. We build 1,000 identical gate systems in PARAMICS and run them at the same time, so that we can get adequate simulation data. Based on experience from the literature, 1,000 replications are enough to reduce the variations so as to get a smooth curve of the queue development.

The second step is to validate the simulation results. Computer simulations are often scenario-specific, so it is necessary to validate the simulation data after collecting them. Here we do it by comparing the steady queue length from simulation with the result from the corresponding stationary queueing model.

The third step is to make a regression analysis on the simulation data for each scenario. First we identify the critical point when the queue formation period ends

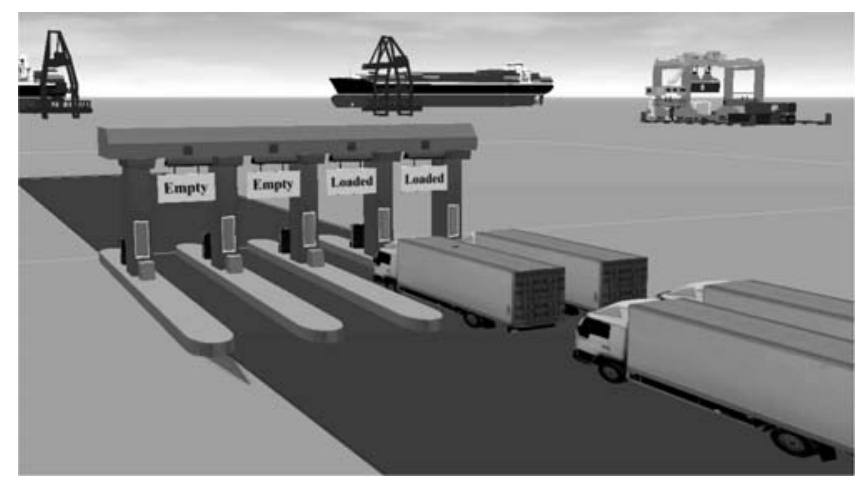

Fig. 2. 3D snapshot of the queue simulation at a terminal gate in PARAMICS.

Developing and running the simulation model of the addressed queueing system

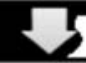

Validating the simulation result with the corresponding stationary queueing model

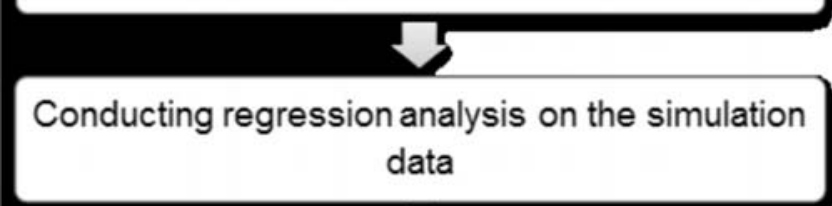

Developing queue length estimation model

Fig. 3. Procedure of developing the queue length estimation model. by calculating the moving average of each time point; then we select the simulation data before the critical point, and analyse the queue formation process by conducting a regression analysis. The aim is to identify a curve that can best fit the simulation data. The fitness can be evaluated with the value of $R^{2}$. Since the simulation data used here are only related to the queueing formation process, the obtained curve also has a valid range from zero to the critical point. After the critical point, the queue will stay in its steady state and its queue length will be equal to the one at the critical point. An example of the curve is

$$
l_{t}=f(t), \quad t \in[0, \text { criticalpoint }] .
$$

where $l_{t}$ is the queue length at time interval $t$. The curves can be used to estimate queue length based on the processing time (queue formation state), or the other way around to project the queue formation state based on the queue length:

In the forth step, based on the identified queue formation curves, we propose a comprehensive model to estimate the truck queue length. The model works in the following way: during the state transition from one time interval to the next, the arrival rate (normally known) determines which curve (parameter) to model, while the queue and the leftover queue length from last interval can be used to project the queue formation state (or time point) with the curve, and as time proceeds one interval the queue length can be estimated following the curve. Suppose a queue at the beginning of time interval $t$ has the queue length $l_{(t-1)}$ and the customer arrival rate is $\lambda_{t}$. The equation

$$
T=f^{-1}\left(l_{t-1}\right)
$$

can project the formation state (or time point) of the queue $T$. The queue length at the end of the time interval $t$ can be estimated with

$$
l_{t}=\min \left(L\left(\lambda_{t}\right), f(T+1)\right) .
$$

which takes the stead queue length $L\left(\lambda_{t}\right)$ when $(T+1)$ exceeds the critical point.

\section{Case study}

5.1. Case description. We select a container terminal in Tianjin Port as a case study to analyse truck queuing. Since the empty and loaded trucks are served in different lanes, we focus only on the gate system for loaded trucks. There are two lanes serving loaded trucks. In general, the truck arrival rate is very dynamic and varies from hour to hour, while the service time is more or less stable with an average of 2.6 minutes and a standard deviation of 0.2 minutes. Truck traffic behaviour is also an important element in the system, because it takes 0.3 minutes on the average for the current truck to leave the service station and for the next truck to get in a position for service. 
Consequently, taking into account both the above times, the system capacity is 40.8 trucks per hour.

Since the service rate is fixed and the customer arrival rate varies between 1 and 40, we design 40 system scenarios to be simulated. The other scenarios in which the customer arrival rate is over the service rate do not need to be simulated, because the queue system utilization rate will be one in these scenarios, and the queue length will increase by the difference between the customer arrival rate and the service rate.

In the next subsection, we introduce step-by-step how the regression model is developed. Then, in the subsequent subsection, we compare the regression model results with those for the existing models.

5.2. Simulation-based regression modelling. We develop a simulation model with PARAMICS for this case terminal gate; a 2D view snapshot of the model is shown in Fig. 4, where each purple block represents one truck. In order to make the queues reach steady state, we run each simulation for a time long enough (24 hours in this case study). Since simulation is driven by stochastic factors, we calculate the average values of 1,000 replications for each scenario. The average queue lengths do not fluctuate strongly and show a clear development trend. Through observation we can find a critical point, where the queue stops increasing and slightly fluctuates within a range. We use this critical point as a boundary to separate the formation process and the steady state. The simulated result can be found in Table 1 .

In order to validate the simulation result, we compare the steady queue length with the one from the $M / G / s$ model, as shown in Fig. 5. We can see the curve of the simulated steady queue length generally fits well the result of the stationary queuing model. The minor difference is probably due to the traffic behaviour modelled in PARAMICS - before joining a queue, a truck normally will speed down to choose a lane. This behaviour is considered a traffic efficiency loss in PARAMICS, but as queuing in the $M / G / s$ model. Despite this minor

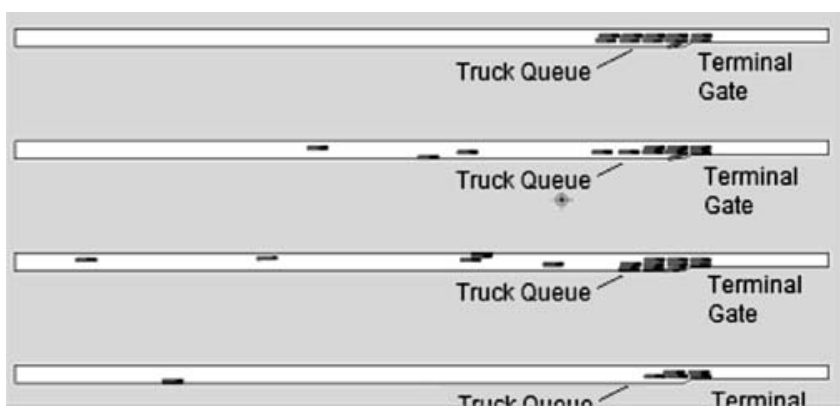

Fig. 4. 2D view snapshot of the simulation model. difference, the good fitting of the two curves indicates that the simulation of the terminal gate system is valid,

The regression model of the natural logarithm curve is

$$
l_{t}=a \ln (t)+b, \quad t \in[0, \text { criticalpoint }],
$$

where $t$ is the time interval and $l_{t}$ is the forming queue length at $t$. From Table 1 we can see that it takes a long time for high arrival rate queues to reach steady state. This finding, as well as the natural logarithm curve, is significant to understand how a queue evolves.

Based on the above results, we develop a comprehensive queuing model. One additional variable is

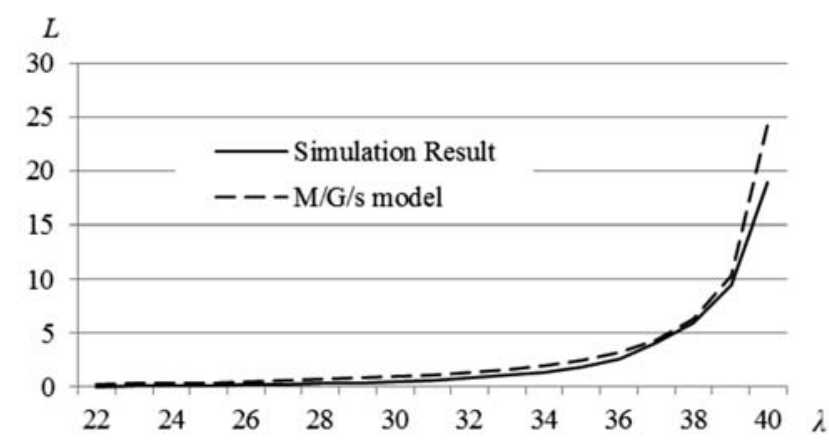

Fig. 5. Comparison of steady queue lengths from simulation and the $M / G / s$ model.

Table 1. Regression result of queue formation process analysis.

\begin{tabular}{|l|l|l|l|l|l|l|}
\hline \multirow{2}{*}{$\lambda$} & \multicolumn{3}{|l|}{$\begin{array}{l}\text { Simulation } \\
\text { result }\end{array}$} & $\begin{array}{l}\text { Regression model } \\
\text { (natural logarithm) }\end{array}$ \\
\cline { 2 - 8 } & $\begin{array}{l}\text { Critical } \\
\text { point } \\
\text { (hour) }\end{array}$ & $L$ & $a$ & $b$ & $R^{2}$ & $\begin{array}{c}\text { Estimated } \\
L\end{array}$ \\
\hline \hline 40 & 24 & 18.90 & 4.89 & 3.15 & 0.94 & 18.69 \\
\hline 39 & 18 & 9.50 & 2.32 & 2.74 & 0.96 & 9.44 \\
\hline 38 & 16 & 6.00 & 1.33 & 2.38 & 0.96 & 6.07 \\
\hline 37 & 15 & 4.10 & 0.95 & 1.54 & 0.97 & 4.11 \\
\hline 36 & 9 & 2.60 & 0.52 & 1.48 & 0.82 & 2.63 \\
\hline 35 & 8 & 1.90 & 0.36 & 1.14 & 0.78 & 1.88 \\
\hline 34 & 6 & 1.30 & 0.28 & 0.82 & 0.77 & 1.33 \\
\hline 33 & 5 & 1.10 & 0.27 & 0.65 & 0.86 & 1.09 \\
\hline 32 & 4 & 0.80 & 0.21 & 0.47 & 0.84 & 0.77 \\
\hline 31 & 4 & 0.60 & 0.15 & 0.39 & 0.76 & 0.59 \\
\hline 30 & 4 & 0.50 & 0.14 & 0.29 & 0.68 & 0.48 \\
\hline 29 & 4 & 0.40 & 0.13 & 0.26 & 0.68 & 0.44 \\
\hline 28 & 4 & 0.30 & 0.07 & 0.17 & 0.69 & 0.26 \\
\hline 27 & 4 & 0.25 & 0.06 & 0.12 & 0.69 & 0.20 \\
\hline 26 & 3 & 0.20 & 0.04 & 0.10 & 0.47 & 0.15 \\
\hline 25 & 3 & 0.15 & 0.04 & 0.09 & 0.47 & 0.14 \\
\hline 24 & 3 & 0.10 & 0.02 & 0.04 & 0.52 & 0.06 \\
\hline 23 & 2 & 0.05 & 0.02 & 0.03 & 0.52 & 0.05 \\
\hline 22 & 1 & 0.05 & 0.01 & 0.02 & 0.61 & 0.02 \\
\hline $21-1$ & 0 & 0 & - & - & - & - \\
\hline & & & & & \\
\hline
\end{tabular}


$\lambda_{t}$, the truck arrival rate at time interval $t$. The proposed model is

$$
\begin{aligned}
& \tau=a\left(\lambda_{t}\right) \ln \left(\exp \left(\frac{l_{t-1}-b\left(\lambda_{t}\right)}{a\left(\lambda_{t}\right)}\right)+1\right)+b\left(\lambda_{t}\right), \\
& l_{t}=\left\{\begin{array}{l}
\min \left\{L\left(\lambda_{t}\right), \tau\right\} \text { if } \lambda_{t} \leq \mu \text { and } l_{t-1} \leq L\left(\lambda_{t}\right), \\
\max \left\{L\left(\lambda_{t}\right), l_{t-1}+\lambda_{t}-\mu\right\} \text { otherwise. }
\end{array}\right.
\end{aligned}
$$

In Eqn. (15), $\exp \left(\left(l_{t-1}-b\left(\lambda_{t}\right)\right) / a\left(\lambda_{t}\right)\right)$ is used to calculate the formation state (or processing time point) of the queue at the previous interval. Adding 1 to the obtained formation state (or processing the time point) and putting it into the natural logarithm function, will get on estimate of the queue length at the current time interval. Because the queue length will step growing in steady state, in Eqn. (16) we choose the smaller one between the steady queue length and the estimated queue length $\tau$. The above described calculation is valid for the conditions in which the arrival rate is not higher than the service rate and the queue length is lower than the steady queue length corresponding to the arrival rate. For the other conditions, we used the lower part of Eqn. (16) to estimate queue length:

5.3. Numerical experiment. In order to compare the proposed model with the existing ones in terms of estimation accuracy, we make a numerical experiment. The existing models include the fluid flow model developed by Chen and Yang (2010), the non-stationary $M(t) / G(t) / 1$ approximation proposed by Chen et al. (2011), and stationary $M / G / s$ model approximation developed by Cosmetatos (1976) as well as the traffic simulation package PARAMICS. In the numerical experiment, it is assumed that the hourly truck arrival rate increases from 35 to 45 during the first 10 hours and decreases to 31 during the rest of the day. Once again, the simulation result obtained from 1,000 replications is considered the exact estimation.

The estimation results of the four models are shown in Fig. 7. We can see that the stationary $M / G / s$

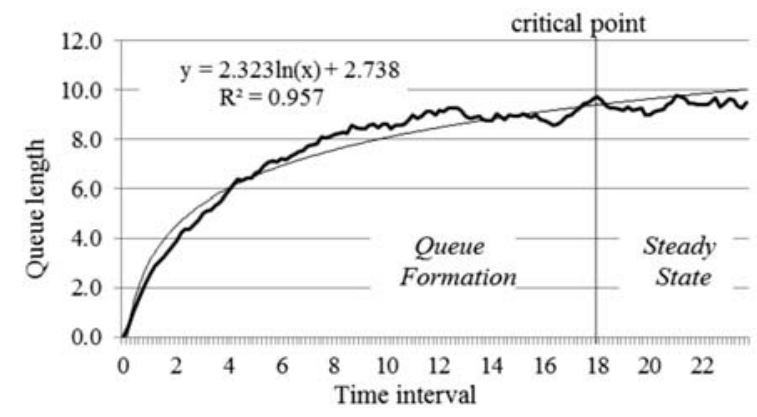

Fig. 6. Queue formation process and its natural logarithm trendline. model apparently overestimates the queue length, and cannot handle the period between 6 a.m., when the demand is over capacity. This overestimation is mainly due to the assumption that the steady state could be reached in a moment. On the other hand, the fluid flow model of Chen and Yang (2010) underestimates the queue length because of neglecting the stochastic queuing phenomenon. The non-stationary $M(t) / G(t) / 1$ approximation of Chen et al. (2011) also significantly overestimates, partially because it assumes the two gate lanes are running separately, which definitely leads to a certain overestimation. The proposed model in this study gives estimations close to the simulation result, although with a bit of underestimation. In summary, the result comparison indicates that the proposed model in this study has high accuracy in estimating the truck queue length at the terminal gate. It also shows that understanding the queue formation process and considering the traffic characteristic are indispensable for a comprehensive analysis of a vehicle queue.

Besides the above accuracy comparison, we also briefly compare the advantages of each model. Simulation is accurate but very time consuming, so it is not suitable for combining with optimization unless in a small-scale problem. The stationary queueing models are not suitable either, because they cannot handle situations where demand is over capacity. Demand management of truck arrivals is a kind of optimization which usually requires a computationally fast model, such as the fluid flow model, the non-stationary $M(t) / G(t) / 1$ approximation or the regression model proposed in this study. Of course, our model is developed based on a large amount of simulation data that was also time consuming to generate, but its advantage in terms of accuracy is outstanding. It can be expected that some approximation for non-stationary $M(t) / G(t) / s(t)$ will be developed in the future, which might be interesting to add into this comparison study.

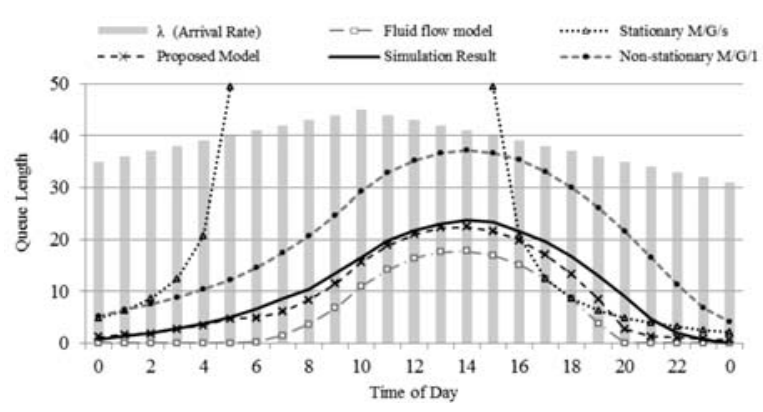

Fig. 7. Estimation result of each model in the numerical experiment. 


\section{Conclusion}

Dealing with truck queues at a gate has become an important task for many major seaports and other big-scale logistic facilities. Regarding truck queue length estimation, it is important to understand the traffic characteristic of the vehicle flow and how a queue generates and evolves. In this paper, we use micro traffic simulation tool to simulate a container terminal gate system and observe the formation process of a truck queue. We find that a queue formation process generally follows a natural logarithm curve and the process takes time especially when the truck arrival rate is high. Based on this finding, we develop a queue length estimation model for truck queues at a container terminal gate. In the experiment, our proposed model performs better than the existing models/approximations, although with slight underestimation compared with the simulation result.

The experiment also shows that stationary queuing models obviously overestimate a queue length, and a fluid flow model neglecting the stochastic queuing phenomenon always underestimates a queue length. Another contribution of this paper is integrating traffic behaviour into queuing analysis, which is a key characteristic of a truck queue. Lastly, the natural logarithm curve of a queue formation process and the proposed queue length estimation model can also be applied in other cases.

\section{Acknowledgment}

This study is funded by an MOE (Ministry of Education in China) Project of Humanities, Social Sciences (11YJC630089) and the National Natural Science Foundation of China (projects 51208079 and 71431001).

\section{References}

Chen, G., Kannan, G., Yang, Z., Choi, T. and Jiang, L. (2013). Terminal appointment system design by non-stationary $M(t) / E k / c(t)$ queueing model and genetic algorithm, International Journal of Production Economics 146(2): 694-703.

Chen, G. and Yang, Z. (2010). Optimizing time windows for managing arrivals of export containers at Chinese container terminals, Maritime Economics and Logistics 2010(12): 111-126.

Chen, X., Zhou, X. and List, G.F. (2011). Using time-varying tolls to optimize truck arrivals at ports, Transportation Research, Part E: Logistics and Transportation Review 47(6): 965-982.

Chydziński, A. and Chróst, Ł. (2011). Analysis of AQM queues with queue size based packet dropping, International Journal of Applied Mathematics and Computer Science 21(3): 567-577, DOI: 10.2478/v10006-011-0045-7.
Cosmetatos, G.P. (1976). Some approximate equilibrium results for the multi-server queue $(M / G / r)$, Operational Research Quarterly 27(3): 615-620.

Davidson, C. (1988). Equilibrium in service industries: An economic application of queuing theory, Journal of Business 61(3): 347-367.

Guan, C. Q. and Liu, R.F. (2009). Modeling maritime container terminal gate congestion, truck waiting cost, and optimization, Transportation Research Record: Journal of the Transportation Research Board 2100(7): 58-67.

Hartmann, S. (2004). Generating scenarios for simulation and optimization of container terminal logistics, OR Spectrum 26(2): 171-192.

Huynh, N., Walton, M.C. and Davis, J. (2004). Finding the number of yard cranes needed to achieve desired truck turn time at maritime container terminals, Transportation Research Record: Journal of the Transportation Research Board 1873(12): 99-108.

Kia, M., Shayan, E. and Ghotb, F. (2002). Investigation of port capacity under a new approach by computer simulation, Computers and Industrial Engineering 42(2): 533-540.

Kim, S. (2009). The toll plaza optimization problem: Design, operations, and strategies, Transportation Research, Part E: Logistics and Transportation Review 45(1): 125-137.

Liu, C.I., Jula, H. and Ioannou, P.A. (2002). Design, simulation, and evaluation of automated container terminals, IEEE Transactions on Intelligent Transportation Systems 3(1): 12-26.

Sacone, S. and Siri, S. (2009). An integrated simulation-optimization framework for the operational planning of seaport container terminals, Mathematical and Computer Modelling of Dynamical Systems 15(3): 275-293.

Shabayek, A. and Yeung, W. (2002). A simulation model for the Kwai Chung container terminals in Hong Kong, European Journal of Operational Research 140(1): 1-11.

Smith, J. (2010). Robustness of state-dependent queues and material handling systems, International Journal of Production Research 48(16): 4631-4663.

Srivatsan, N. and Kempf, K. (1995). Effective modeling of factory throughput times, 17th IEEE/CPMT International Electronics Manufacturing Technology Symposium, Omiya, Japan, pp. 377-383.

Taniguchi, E., Noritake, M., Yamada, T. and Izumitani, T. (1999). Optimal size and location planning of public logistics terminals, Transportation Research, Part E: Logistics and Transportation Review 35(3): 207-222.

Wu, J., Abbas-Turki, A. and Perronnet, F. (2013). Cooperative driving at isolated intersections based on the optimal minimization of the maximum exit time, International Journal of Applied Mathematics and Computer Science 23(4): 773-785, DOI: 10.2478/amcs-2013-0058.

Xie, Y., Chowdhury, M., Bhavsar, P. and Zhou, Y. (2012). An integrated modeling approach for facilitating emission estimations of alternative fueled vehicles, Transportation Research, Part D: Transport and Environment 17(1): 15-20. 
Yang, C.H., Choi, Y.S. and Ha, T.Y. (2004). Simulation-based performance evaluation of transport vehicles at automated container terminals, OR Spectrum 26(2): 149-170.

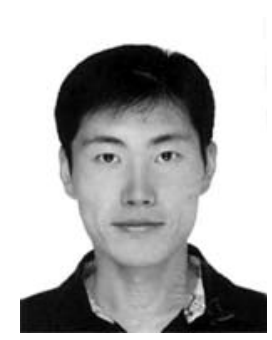

Gang Chen is a Ph.D. student at the Transport Management College of Dalian Maritime University (China), working on the topic of marine terminal operation modelling with the focus on the congestion issues. His current research interests are discrete event simulation, queueing theory, shipping and logistics, and agent-based simulation.

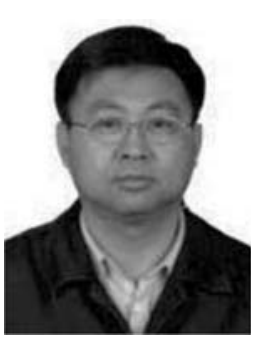

Zhongzhen Yang received his $\mathrm{Ph} . \mathrm{D}$. from Nagoya University in Japan. His research interests include traffic demand modeling, transportation planning, transport network analysis, shipping and logistics, and port operations. He has written two books and published over 90 papers in refereed journals. Currently he is a professor at the Transport Management College of Dalian Maritime University, China.

Received: 1 August 2013

Revised: 13 December 2013

Re-revised: 18 February 2014 